\title{
1 IgA Potentiates NETosis in Response to Viral Infection
}

3 Hannah D. Stacey ${ }^{1,2,3}$, Diana Golubeva ${ }^{1,2,3}$, Alyssa Posca ${ }^{1,2,3}$, Jann C. Ang ${ }^{1,2,3}$, Kyle E.

4 Novakowski $^{1,2,4}$, Muhammad Atif Zahoor ${ }^{2,4, a}$, Charu Kaushic ${ }^{2,4}$, Ewa Cairns ${ }^{5,6}$, Dawn M. E.

5 Bowdish $^{1,2,4}$, Caitlin E. Mullarkey ${ }^{1}$ and Matthew S. Miller ${ }^{1,2,3^{*}}$

$7{ }^{1}$ Michael G. DeGroote Institute for Infectious Diseases Research, McMaster University, 8 Hamilton, ON, Canada

$9{ }^{2}$ McMaster Immunology Research Centre, McMaster University, Hamilton, ON, Canada

$10{ }^{3}$ Department of Biochemistry and Biomedical Sciences, McMaster University, Hamilton, ON,

11 Canada

$12{ }^{4}$ Department of Medicine, McMaster University, Hamilton, ON, Canada

13 Department of Microbiology and Immunology, Schulich School of Medicine and Dentistry,

14 Western University, London, ON, Canada

${ }^{6}$ Department of Medicine, Division of Rheumatology, Schulich School of Medicine and Dentistry, Western University, London, ON, Canada

17 a Current address: Toronto Center for Liver Disease, Toronto General Hospital Research, Institute, MaRS-Princess Margaret Cancer Research Tower 10-401, University Health Network, 101-College St. Toronto, ON, Canada

*Corresponding author: McMaster University, 1280 Main St. W, Hamilton, ON, Canada, L8S

$4 \mathrm{~K} 1$

Email: mmiller@mcmaster.ca

KEYWORDS: NETosis; NETs; neutrophils; IgA; Fc alpha receptor; influenza virus; broadly- 


\section{ABSTRACT}

43

$44 \operatorname{Ig} \mathrm{A}$ is the second most abundant antibody present in circulation and is enriched at mucosal

45 surfaces. As such, IgA plays a key role in protection against a variety of mucosal pathogens,

46 including viruses. In addition to neutralizing viruses directly, IgA can also stimulate Fc-dependent

47 effector functions via engagement of Fc alpha receptors (Fc $\alpha \mathrm{RI})$ expressed on the surface of certain

48 immune effector cells. Neutrophils are the most abundant leukocyte, express FcaRI, and are often

49 the first to respond to sites of injury and infection. Here, we describe a novel function for IgA:virus

50 immune complexes (ICs) during viral infections. We show that IgA:virus ICs potentiate NETosis

51 - the programmed cell death pathway through which neutrophils release neutrophil extracellular

52 traps (NETs). Mechanistically, IgA:virus ICs potentiated a suicidal NETosis pathway via

53 engagement of FcaRI on neutrophils through a toll-like receptor (TLR)-independent, NADPH

54 oxidase complex-dependent pathway. NETs also were capable of trapping and inactivating viruses,

55 consistent with an antiviral function. 


\section{5}

76

77

78

79

80

81

82

83

84

92 injury ${ }^{5}$.

93 The generation of NETs was first described by the Zychlinsky laboratory in 2004 as an

94 antibacterial effector mechanism ${ }^{6}$. NETs produced via a specialized form of programmed cell

95 dealth called "NETosis" and are composed primarily of decondensed chromatin studded with

96 antimicrobial proteins. Extensive work by many laboratories has since demonstrated that NETs

97 can have not only protective, but also pathogenic consequences in infections and many other

98 diseases ${ }^{4}$. The understanding of how NETs influence viral infections continues to evolve. In the 
context of Chikungunya virus and poxvirus, NETs were capable of trapping virus and controlling infection in a mouse models of disease ${ }^{7,8}$. Likewise, NETs have been shown to trap and inactivate HIV $^{9}$. However, NETs have also been described to exacerbate disease in the context of Dengue virus, rhinovirus, respiratory syncytial virus, influenza virus, and most recently, SARS-CoV-2 infection ${ }^{10-16}$. Thus, the overall impact of NETs during a viral infection must be interpreted

104 carefully in conjunction with other infection parameters.

105 Recently, Fc-dependent effector functions have been shown to play a central role in the protection 106 conferred by broadly-neutralizing antibodies (bnAbs) that bind to the hemagglutinin (HA) stalk 107 domain of IAV ${ }^{17-19}$. However, these studies have only been performed in the context of 108 monoclonal IgG antibodies. Elicitation of bnAbs is now the goal of several "universal" influenza 109 virus vaccine candidates, including "chimeric" HA vaccines that were recently tested in a Phase I 110 clinical trial ${ }^{20}$. Antibody-dependent cellular cytotoxicity (ADCC) may also augment protection 111 mediated by human immunodeficiency virus (HIV)-neutralizing antibodies ${ }^{21}$. However, despite 112 the fact that both IAV and HIV are mucosal pathogens - almost nothing is known about the 113 contribution of IgA-mediated Fc-dependent effector functions during infection. This is due, in 114 large part, to the fact that mice do not express an FcaR homolog which presents significant 115 challenges for assessing the contributions of IgA to outcomes in vivo ${ }^{1}$.

116 Here, we show that IgA:virus ICs potentiated NETosis through FcaRI signaling on neutrophils. 117 This potentiation was not virus-specific, and could be observed for IAV, HIV, SARS-CoV-2 and 118 extended to IgA ICs generated with antibodies/autoantigens from RA patients. In contrast to 119 NETosis stimulated by virus directly, IgA:virus ICs stimulated suicidal NETosis that was 120 independent of TLR signaling. Finally, viruses were trapped and inactivated in NETs, suggesting 121 a protective role in vivo when properly regulated. 


\section{RESULTS}

\section{IgA:IAV immune complexes stimulate NETosis}

125 Historically, antibodies have been thought to mediate protection against influenza viruses 126 primarily by binding to the HA head domain and blocking interaction between the receptor binding

127 site on HA and sialic acids on the surface of host cells. However, more recently it has become 128 clear that bnAbs that bind to the HA stalk domain mediate protection in vivo primarily by 129 elicitation of Fc-dependent effector functions ${ }^{17-19}$. Antigen-specific IgA antibodies have been 130 shown to neutralize IAV, but relatively little is known about IgA-mediated Fc-dependent effector 131 functions during IAV infection ${ }^{22}$. Neutrophils are the most abundant leukocyte and are among the

132 first to respond during IAV infection ${ }^{23}$. Neutrophils also express FcaRI, and we have previously

133 shown that IgA:IAV ICs stimulate ROS production in neutrophils; however, unlike IgG:influenza

134 virus ICs, this could not be fully inhibited by cytochalasin D - indicating that IgA-mediated ROS 135 production was not due to antibody-dependent cellular phagocytosis (ADCP) ${ }^{24}$. To determine

136 whether IgA was capable of potentiating NETosis upon binding IAV, neutrophils were exposed to 137 antibody:IAV ICs composed of polyclonal (monomeric) IgA or IgG from the peripheral blood of 138 donors previously vaccinated with seasonal influenza vaccines containing the 139 A/California/04/2009 (Cal/09) H1N1 component. Phorbol 12-myrisate13-acetate (PMA), a 140 potent inducer of NETosis, was used as a positive control ${ }^{25}$. IgA:IAV ICs stimulated significantly 141 higher levels of NETosis than antibodies or virus alone, whereas IgG:IAV ICs did not induce 142 NETosis above background levels (Fig. 1A, B).

143 In the context of IgG, bnAbs that bind to the stalk domain have been shown to potently elicit Fc144 dependent effector functions, whereas antibodies that bind to the HA head domain and exhibit 
145 hemagglutination inhibiting (HAI) activity do not. This is because HA stalk-binding bnAbs allow

146 for a two points of contact between target and effector cells ${ }^{26,27}$. To determine whether broadly-

147 neutralizing IgA:IAV ICs are primarily responsible for the induction of NETosis observed in the

148 context of IAV-specific polyclonal IgA, we used a panel of previously-described monoclonal

149 antibodies that bind to neutralizing epitopes on either the HA head or stalk domains ${ }^{28-30}$. The

150 antibody KB2 binds to the HA stalk domain of H1 viruses, while 29E3 is specific to the HA head

151 domain of $\mathrm{Cal} / 09^{31}$. When human neutrophils were incubated with ICs containing a IAV:IgA

152 stalk-binding antibody (KB2), significant induction of NETosis was observed following 3-hour

153 stimulation (Fig. 1C). In contrast, NETosis was not induced by IgG:IAV ICs, or by antibodies or

154 virus alone (Fig. 1C). All ICs generated with a HA head-binding antibody (29E3) failed to induce

155 NETosis (Fig. 1D).

156 In blood, IgA co-circulates with other antibodies, including IgG, which signals through distinct

157 FcRs (FcyRs) and can also induce NETosis ${ }^{32}$. Mixed ICs composed of IgG/IgA:HIV have also

158 been shown to act cooperatively to stimulate ADCC by monocytes ${ }^{33}$. We therefore tested whether

159 mixed ICs composed of IAV bound by $\operatorname{IgA}$ and IgG together would influence the magnitude of

160 NETosis induction relative to IgA alone. When ICs were generated with a 1:1 ratio of IgG:IgA,

161 the magnitude of NETosis induction was similar to IgA alone (Fig. 1E). In serum, IgG is

162 significantly more abundant than IgA (approx. 4:1 to 10:1). Thus, to recapitulate the physiological

163 stoichiometry of IgG:IgA, we purified each immunoglobulin from serum of matched donors, and

164 then recombined them at their natural physiological ratio. Here again, the magnitude of NETosis

165 observed in mixed $\operatorname{IgG}: \operatorname{Ig}$ A immune complexes was similar to $\operatorname{Ig}$ A alone, indicating that $\operatorname{IgG}$ does

166 not potentiate IgA-mediated NETosis, nor does it interfere with the ability of IgA to stimulate 
167 NETosis (Fig. 1F). Taken together, these results demonstrate that IgA:virus ICs stimulate 168 neutrophils to undergo NETosis.

170 IgA-mediated NETosis is not an IAV-specific phenomenon

171 NETs have been observed in the context of many other infections, including those caused by

172 SARS-CoV-2 and HIV. In these studies, viruses were presumed to stimulate NETosis directly

$17314,16,34,35$. We thus performed an experiment to test the amount of virus needed to stimulate NETosis

174 independent of FcaR signaling. Neutrophils were stimulated with increasing concentrations of

175 purified lentiviruses pseudotyped with the SARS-CoV-2 spike protein. A significant elevation in

176 NETosis was observed when neutrophils were exposed to 0.05 and $0.2 \mathrm{mg} / \mathrm{mL}$ of purified virus

177 (Fig. 2A). We then purified IgA from convalescence serum of a SARS-CoV-2 infected individual,

178 and a SARS-CoV-2 naïve individual, incubated them with sub-stimulatory concentrations $(0.0125$

$179 \mathrm{mg} / \mathrm{mL}$ ) of spike pseudotyped lentiviruses to allow for IC formation, and then incubated these

180 mixtures with primary human neutrophils from healthy donors. As we observed in the context of

181 IAV, IgA:virus IC generated with IgA purified from SARS-CoV-2 convalescence serum was

182 capable of stimulating NETosis, whereas pseudovirus:IgA mixtures from naïve serum was not (Fig

183 2B). These results confirm that IgA:virus ICs more potently stimulate NETosis when compared to

184 virus alone, and that ICs are required for this potentiation, since IgA from seronegative individuals

185 did not significantly induce NETosis when mixed with pseudotyped lentivirus.

186 We also incubated neutrophils with antibody:HIV ICs which contained HIV-specific IgA isolated

187 from the serum of HIV+ individuals. Following stimulation, a significant increase in NETosis was

188 observed in cells treated with anti-HIV IgA containing ICs (Fig. 2C). Background levels of 
NETosis wcre observed when cells were treated with either IgA or virus alone. These findings

190

191

192

193

194

195

196

197

198

199

200 201 IgA:autoantigens.

202

203

204

205

206

207

208

209

210

\section{of TLR signaling}

demonstrate that $\operatorname{Ig} \mathrm{A}$ induced NETosis likely happens in the context of many viral infections.

NETs have also been implicated in the pathogenesis of a variety of autoimmune conditions, including rheumatoid arthritis (RA) where they serve as a source of autoantigen ${ }^{36,37}$. Patients with autoimmune diseases commonly have autoantibodies against NET elements such as histones, DNA, and neutrophil elastase. Here, neutrophils were stimulated with Ab:autoantigen ICs composed of IgA or IgG purified from the serum of RA patients or healthy donors, and recombinant citrullinated human fibrinogen, a common autoantigen in RA ${ }^{38}$. Induction of NETosis was observed in neutrophils stimulated with IgA:citrullinated fibrinogen immune complexes from RA patients, but not in those stimulated with IgG-containing ICs or ICs generated with antibodies from healthy donors (Fig. 2D). Together, these data demonstrate that potentiation of NETosis is a common property of virus: $\operatorname{IgA}$ immune complexes, as well as ICs composed of

\section{Induction of NETosis by IgA immune complexes is dependent on FcaRI and independent}

We next assessed whether salivary $\operatorname{IgA}(\operatorname{sg} A)$ was capable of inducing NETosis. Whereas monomeric $\operatorname{IgA}(\mathrm{mIgA})$ is found predominantly in circulation, secretory $\operatorname{IgA}$ is enriched at mucosal surfaces and is generally regarded as an anti-inflammatory antibody. sIgA from saliva and serum-derived mIgA was purified from matched vaccinated donors used to generate ICs with IAV. ICs containing sIgA did not potentiate NETosis, whereas serum-derived mIgA from the same donors was capable of eliciting NETosis, as we had observed previously (Fig. 3A). These results 
211 are consistent with previous studies that have demonstrated that the secretory component sterically

212 blocks binding of secretory IgA to Fc $\alpha$ RI (CD89) ${ }^{39}$.

213 Given the observation that sIgA:IAV ICs failed to induce NETosis, we investigated whether

214 mIgA-mediated NETosis was dependent on engagement of FcaRI (CD89). To this end, neutrophils

215 were incubated with a blocking monoclonal anti-CD89 antibody prior to stimulation with

216 IgG:virus or IgA:virus ICs. Blocking with anti-CD89 abrogated induction of NETosis following

217 stimulation with IgA ICs (Fig. 3B), confirming that engagement of Fc $\alpha$ RI is required for IgA:virus

218 IC-mediated induction of NETosis.

219 TLR8 activation has been shown to shift neutrophils from phagocytosis to NETosis in the context

220 of IgG IC-mediated NETosis via Fc $\gamma$ RIIA signaling ${ }^{32}$. We thus set out to determine whether TLR

221 signaling was required for IgA-mediated NETosis induction. TLR8 senses single-stranded RNA

222 and is an important pattern-recognition receptor during RNA virus infection ${ }^{40}$. Since IAV particles

223 contain RNA, we elected to use a system free from TLR7/8 ligands. To this end, polystyrene beads

224 (roughly equal in number to IAV particles used in previous experiments) were coated with protein

$225 \mathrm{~L}$ and polyclonal IgA. Protein L binds to the $\kappa$ light chain of antibodies, leaving the antibody Fc

226 region capable of interacting with FcRs on the cell surface. Following stimulation, IgA:bead ICs

227 induced significant NETosis relative to beads alone (Fig. 3C). This suggests that unlike IgG IC-

228 mediated NETosis, IgA IC-mediated NETosis is likely independent of TLR signaling.

229 Neutrophils are professional phagocytes, and ADCP is one of the many Fc-mediated effector

230 functions that contribute to their defense against pathogens ${ }^{24}$. To directly measure whether $\operatorname{IgA}$

231 ICs induced phagocytosis, fluorescent, protein L-coated polystyrene beads were complexed with

$232 \operatorname{IgA}$ or IgG prior to incubation with neutrophils. After incubation with beads, cells were washed 233 extensively to remove any beads that had not been phagocytosed. Significantly greater bead uptake 
was recorded for neutrophils that were exposed to the IgG-opsonized beads compared to those coated with IgA, which actually inhibited phagocytosis relative to protein L-coated control beads (Fig. 3D). This further demonstrates that endosomal TLR activation by viral pathogen-associated molecular patterns (PAMPs) are not required for the potentiation of NETosis by IgA:virus ICs. As further confirmation, instead of using soluble ICs as had been done in previous experiments, IC's were immobilized on glass coverslips. Consistent with all experiments that had been performed using soluble ICs, significantly higher levels of NETosis were observed when neutrophils were

241 incubated with immobilized IgA:virus ICs relative to immobilized IgG:virus containing ICs (Fig.

242 3E). Combined, these data suggest that phagocytosis is not required for IgA IC-mediated 243 stimulation of NETosis.

\section{IgA ICs stimulate NADPH oxidase complex (NOX)-dependent suicidal NETosis}

246 The most common and well-characterized type of NETosis is called "suicidal NETosis", which

247 results in the death of the cell. More recently, other types of NETosis have been described,

248 including "vital” NETosis ${ }^{41}$. Suicidal NETosis requires ROS production and occurs between 1-3

249 hours after stimulation, while vital NETosis does not require the generation of ROS and occurs

250 between 5 and 60 minutes after stimulation ${ }^{41}$. To determine whether IgA:virus IC-induced

251 NETosis was vital or suicidal, we first performed a time-course experiment following stimulation

252 with PMA, a well characterized stimulant of suicidal/ROS-dependent NETosis, or IgA:IAV ICs

253 for 30, 90 or 180 minutes (Fig. 4A). A significant increase in NETosis was observed following

254 incubation with IgA:IAV ICs for 180 minutes, consistent with suicidal NETosis. Unsurprisingly,

255 PMA - a far more potent stimulant, significantly induced NETosis beginning at 90 min after

256 stimulation (Fig. 4A). Conversely, to inhibit the production of ROS, a small molecule inhibitor of 
the NOX complex, diphenyleneiodonium chloride (DPI), was pre-incubated with neutrophils prior to stimulation with IgA:IAV ICs. DPI completely inhibited NETosis induced by IgA ICs (Fig. 4B). Together, these observations demonstrate that IgA:virus ICs stimulate suicidal NET release in a NOX-dependent manner.

\section{Virus particles are trapped and inactivated by NETs}

263 In the context of bacterial infections, NETs exert antimicrobial activity by trapping and killing

264 bacteria with antimicrobial effector proteins associated with NETs. We thus set out to determine

265 whether NETs were similarly capable of trapping and inactivating virus. Neutrophils were either

266 left unstimulated or were treated with PMA to induce suicidal NETosis (virus containing ICs were

267 not used to avoid the confounding issue of having viruses present during induction of NETosis).

268 IAV was then incubated in wells of stimulated or unstimulated neutrophils, and unbound virus was

269 washed away. Using immunofluorescence microscopy, we observed that IAV particles become

270 trapped in NETs induced following stimulation with PMA (Fig. 4C). Using ImageJ software, we

271 quantified GFP pixel density and normalized this to the number of cells (and/or NETs) per field.

272 Consistent with the stark visual contrast observed in the images, significantly more virus was

273 associated with PMA-stimulated neutrophils that had undergone NETosis than unstimulated

274 neutrophils (Fig. 4D).

275 To test whether IAV was inactivated after being trapped in NETs, we used an mNeon reporter

276 virus $^{42}$. IAV-mNeon was incubated with unstimulated neutrophils, PMA-stimulated neutrophils

277 that had undergone NETosis, or PMA-stimulated neutrophils treated with DNase to digest NETs.

278 DNase digestion specifically allowed us to test whether being trapped in a NET was necessary for

279 inactivation, or whether factors released by neutrophils during NETosis were alone sufficient to 
280 inactivate IAV (Supplementary Fig. 1, Fig. 4E). After 3h or 6h incubation, viral media was

281 collected from all wells incubated on Madin Darby Canine Kidney cells (MDCKs) to quantify

282 remaining infectious virus. Incubation of virus with PMA-stimulated neutrophils that had

283 undergone NETosis significantly reduced infectivity after $3 \mathrm{~h}$ and $6 \mathrm{~h}$ incubation. Interestingly,

284 digestion of NETs produced by PMA-simulated cells with DNase prior to addition of virus had no

285 significant impact of infectivity - suggesting that physical contact with NETs is required for

286 inactivation and that soluble factors released during the process of NETosis alone are not sufficient

287 to mediated inactivation (Fig. 4E). Taken together, these data demonstrate the viruses can be

288 trapped and inactivated by NETs.

\section{DISCUSSION}

291 NETosis has been most extensively studied as an anti-pathogen immune response in the context

292 of bacterial infections ${ }^{43}$. However, accumulating evidence suggests that NETs have antiviral

293 activity, but can also contribute to the pathogenesis of viral disease in certain circumstances ${ }^{8-}$

$29411,44,45$. While pathogens like viruses and bacteria can trigger NETosis directly as an innate immune

295 mechanism, there is also an important intersection of neutrophils/NETs and the adaptive immune

296 response, since neutrophils express Fc receptors capable of recognizing both soluble ICs and

297 antibody-bound cells. Here, we show that IgA significantly lowers the amount of virus required to 298 trigger NETosis.

299 In the context of IgG, immobilized ICs have been reported to stimulate NETosis via Fc $\gamma$ RIIA.

300 Soluble ICs were primarily phagocytosed, but could be shifted to stimulate NETosis upon TLR7/8

301 activation, which resulted in furin-mediated cleavage and shedding of the Fc $\gamma$ RIIA N-terminus -

302 inhibiting further phagocytosis ${ }^{32}$. We observed that IgA ICs did not simulate phagocytosis, but 
rather preferentially induced NETosis, even in the absence of TLR activation. While IgG ICs could

304 stimulate NETosis, the induction of NETosis was notably more pronounced upon stimulation of

305 neutrophils with IgA ICs.

306 In the context of IAV, bnAbs that bind to the conserved HA stalk domain have become a major

307 focus for the development of "universal" influenza virus vaccines and monoclonal antibody

308 prophylactics/therapeutics. Although bnAbs are relatively weak neutralizers of IAV, they confer

309 protection in vivo by potent induction of Fc-dependent effector functions ${ }^{17-19,22,24,26,27}$. The ability

310 of bnAbs to elicit potent effector functions (relative to conventional neutralizing antibodies that

311 bind to the HA head domain) relies on a unique reciprocal contact model whereby Fc receptors of

312 immune effector cells bind to the Fc domain of bnAbs bound to HA on target cells, while HA

313 expressed on target cells in turn binds to sialic acid residues of the effector cell ${ }^{26,27}$. However,

314 almost everything that is known about the function of bnAbs has been studied in the context of

315 IgG. Of the other immunoglobulin isotypes, IgA plays a particularly important role in protection

316 against mucosal viruses. Indeed, local IgA responses correlate with protection offered by live-

317 attenuated influenza virus vaccines ${ }^{46-48}$. A recent Phase I trial of a chimeric HA universal vaccine

318 candidate reported potent induction of $\operatorname{IgA}$ bnAbs after vaccination - further highlighting the

319 urgent need to understand how antibodies of this isotype contribute to protection ${ }^{20,49}$. Here, we

320 show that consistent with prior studies, bnAbs are primarily responsible for induction of Fc $\alpha$ RI-

321 dependent NETosis, likely because these antibodies also promote the reciprocal binding events

322 between $\operatorname{IgA}: F c \alpha R I$ and HA:sialic acid described above.

323 Importantly, the ability of IgA ICs to potentiate NETosis was widespread across several different

324 viruses including IAV, lentiviruses pseudotyped with SARS-CoV-2 S protein, and HIV. Indeed,

325 this phenomenon could also be recapitulated with IgA-coated beads and extended beyond the 
326 context of infectious diseases to ICs composed of IgA from RA patients in complex with

327 citrullinated fibrinogen - a common RA autoantigen. Our findings support previous work from the

328 van Egmond laboratory demonstrating that IgA ICs isolated from synovial fluid of RA patients

329 also induce NETosis ${ }^{36}$. Previous work by our group has shown that upon exposure to IgG:IAV

330 ICs, neutrophils undergo ADCP and potently induce ROS. Inhibition of phagocytosis with

331 cytochalasin D almost completely abolished ROS induction by IgG:IAV ICs. In contrast, IgA:IAV

332 ICs were able to stimulate ROS even when phagocytosis was inhibited ${ }^{24}$. Those observations are

333 in line with the data presented herein showing that IgA:ICs induced neutrophils to undergo ROS-

334 dependent suicidal NETosis in a phagocytosis-independent manner.

335 In serum, $\operatorname{IgA}$ is present at concentration of $\sim 82-624 \mathrm{mg} / \mathrm{dL}$, whereas $\mathrm{IgG}$ is found at $\sim 694-1803$

$336 \mathrm{mg} / \mathrm{dl}^{50}$. In the context of HIV, mixed IgG/IgA:HIV ICs generated using the gp41-specific bnAb

337 2F5 cooperatively triggered ADCC of HIV-infected cells by monocytes, but did not act

338 cooperatively to induce ADCP ${ }^{33,51}$. Likewise, we observed no cooperativity in the induction of

339 NETosis when IgA and IgG were combined at a 1:1 ratio, or at physiological ratios. These results

340 suggest that signaling downstream of Fc $\gamma$ Rs and FcaRI lead to distinct effector outcomes in

341 monocytes and neutrophils.

342 While ICs composed of serum-derived IgA and monomeric monoclonal IgA could both potentiate

343 NETosis, secretory IgA purified from saliva could not. This is consistent with prior studies that

344 have demonstrated that the secretory component sterically interferes with binding to Fc $\alpha$ RI and

345 suggests that IgA-stimulated NETosis is unlikely to occur in the airways where secretory $\operatorname{IgA}$ is

346 enriched, but instead would be expected to take place primarily in tissues and vasculature ${ }^{52}$.

347 The data presented here demonstrate that NETs can both trap and inactivate virus. This suggests

348 that they may have a protective antiviral function. We speculate that in individuals who lack virus- 
349 specific IgA, the high concentrations of virus needed to stimulate NETosis might exacerbate

350 inflammation and potentiate disease, as has been observed for those with COVID-19 ${ }^{14-16,35}$.

351 However, individuals with pre-existing immunity - such as that conferred by vaccines - low levels

352 of IgA-induced NETosis might help to trap and inactivate virus early in infection, thereby limited

353 virus spread and progression to severe disease.

354 In summary, we report a new antiviral effector function mediated by virus:IgA ICs. The

355 mechanism through which virus:IgA ICs stimulate NETosis is distinct from, and considerably

356 more potent than virus alone. Since mice do not express an FcaR, it will be important to develop

357 alternative models for in vivo studies to determine when IgA:virus IC-mediated NETosis may be

358 protective, and when it may exacerbate disease.

MATERIALS AND METHODS

\section{Human Serum and Blood Samples}

362 Human blood samples used to isolate serum antibodies were obtained with permission from

363 consenting male and female IAV-vaccinated donors, SARS-CoV-2 infected donors, HIV-

364 positive individuals, and RA patients. Human blood for neutrophil isolations were collected with

365 permission from consenting healthy male and female donors. All protocols involving human

366 samples were approved by the Hamilton Integrated Research Ethics Board and the Western

367 Research Ethics Board. Blood was collected into Ethylenediamine tetra-acetic acid (EDTA)

368 coated tubes (BD Vacutainer).

\section{Neutrophil Isolation}

370 Neutrophils were isolated from the peripheral blood of healthy male and female donors by

371 density gradient centrifugation as described previously ${ }^{24}$. Briefly, $3 \mathrm{~mL}$ of room temperature 
372 Histopaque 1119 (Sigma-Aldrich) was added to a $15 \mathrm{~mL}$ falcon tube, followed by gentle addition

373 of $3 \mathrm{~mL}$ of Histopaque 1077 (Sigma- Aldrich). $6 \mathrm{~mL}$ of blood was layered on top and samples

374 were centrifuged at $930 \mathrm{x}$ g for 30 minutes at room temperature (RT) with no deceleration in an

375 Allegra X-12R centrifuge (Beckman Coulter). The neutrophil layer was collected between the

376 Histopaque layers and diluted in $4^{\circ} \mathrm{C}$ PMN buffer $(0.5 \%$ BSA, 0.3 mM EDTA in Hank's

377 balanced salt solution (Sigma-Aldrich)) to a total volume of $50 \mathrm{~mL}$. PMNs were then centrifuged

378 at $450 \mathrm{x}$ g for 5 minutes at RT. The Supernatant was discarded, and the cell pellet re-suspended

379 by flicking the tube. To lyse red blood cells, $3 \mathrm{~mL}$ of ACK (ammonium-chloride-potassium)

380 lysis buffer $\left(8.3 \mathrm{~g} / \mathrm{L} \mathrm{NH}_{4} \mathrm{Cl}, 1 \mathrm{~g} / \mathrm{L} \mathrm{KHCO}_{3}, 0.05 \mathrm{mM}\right.$ EDTA, in sterile distilled $\left.\mathrm{H}_{2} \mathrm{O}\right)$ was added

381 to the PMNs and incubated for 3 minutes with agitation every 30 seconds. The PMNs were

382 diluted in $30 \mathrm{~mL}$ of PMN buffer and centrifuged at $450 \mathrm{x} \mathrm{g}$ for 5 minutes at RT, followed by one

383 additional wash.

\section{Antibody Purification}

385 Heat-inactivated human serum was diluted 1:10 in phosphate buffered saline (PBS) and applied

386 to a gravity polypropylene flow column (Qiagen) containing $1 \mathrm{~mL}$ of Protein G-sepharose resin

387 (Invitrogen) to purify IgG. Flow through sera was then applied to a gravity flow column

388 containing $1 \mathrm{~mL}$ Peptide M-sepharose resin (InvivoGen) to purify IgA. Columns were washed

389 with two column volumes of PBS. IgG and $\operatorname{IgA}$ were eluted with $0.1 \mathrm{M}$ glycine- $\mathrm{HCl}$ buffer (pH

390 2.7) into $2 \mathrm{M}$ Tris- $\mathrm{HCl}$ neutralizing buffer ( $\mathrm{pH} 10)$. Antibodies were concentrated and re-

391 suspended in PBS using 30 kDa cutoff Macrosep Advanced Centrifugal Devices (Pall

392 Corporation). To purify monoclonal antibodies, clarified cell culture supernatants were applied

393 directly to Protein G-sepharose columns prior to washing and elution.

\section{Monoclonal Antibodies}


395 The variable light and heavy chain sequences of KB2 and $29 \mathrm{E} 3$ antibodies ${ }^{30,53}$ were cloned into

396 cloned into pFuse vectors (pFUSE-hIgG1-Fc2 and pFUSE2ss-CLIg-hK, Invivogen). KB2 binds

397 to the stalk domain of H1 viruses, while 29E3 antibody is specific to the head domain of

398 A/California/04/09 (Cal/09). HEK293T cells co-transfected with pFUSE plasmids according to

399 manufacturer's recommendations and were subsequently purified from supernantants using

400 Protein G-sepharose columns, as described above.

401 Cells and Viruses

402 Madin Darby Canine Kidney (MDCK) cell were grown in Dulbecco modified Eagle medium

403 (DMEM) containing 10\% fetal bovine serum (FBS) (Gibco), 2 mM L- and 100 U/mL penicillin-

404 streptomycin (Thermo Fisher). At 100\% confluency MDCK cells were infected for one hour

405 with A/California/04/2009 H1N1 (kind gift of Dr. Peter Palese, Icahn School of Medicine at

406 Mount Sinai, New York, NY) in 1x minimum essential medium (MEM, Sigma Aldrich)

407 supplemented with 2 mM L-glutamine, 0.24\% sodium bicarbonate, 20 mM HEPES (4-(2-

408 hydroxyethyl)-1-piperazineethanesulfonic acid), MEM amino acids solution (Sigma Aldrich),

409 MEM vitamins solution (Sigma Aldrich), $100 \mathrm{U} / \mathrm{mL}$ penicillin-streptomycin (Thermo Fisher),

410 and $0.42 \%$ bovine serum albumin (Sigma Aldrich). Cells were then washed with PBS and media

411 was replaced. Cells were left for 72 hours and supernatant was collected. A/ Puerto Rico/8/1934/

412 H1N1-mNeon (PR8-mNeon, which was a kind gift from the laboratory of Dr. Nicholas Heaton

413 (Duke University, Durham, NC) ${ }^{42}$, was propagated in 10- day- old embryonated chicken eggs as

414 per standard protocols (WHO, 2011).

\section{Influenza Virus Purification}

416 Clarified supernatants from IAV-infected MDCK cells were layered on top of $8 \mathrm{~mL} 20 \%$ sucrose

417 (Bioshop) in NTE buffer (0.5 M NaCl, $10 \mathrm{mM}$ Tris-HCl, $1 \mathrm{mM}$ EDTA, pH 7.5) inside Ultra- 
418 Clear Ultracentrifuge tubes (Beckman Coulter). Samples were spun at 76,650 x g for 2 hours at

$419 \quad 4^{\circ} \mathrm{C}$ inside a SW 32i rotor using an Optima L-90K Ultracentrifuge (Beckman Coulter). Purified

420 virus was quantified using a bicinchoninic acid assay (BCA) Protein Assay Kit (Pierce

421 Biotechnology) according to the manufacturer's instructions, and by hemagglutination assay.

\section{Psuedotyped Lentivirus Production}

423 HIV-1 X4 gp120 pseudotyped lentiviruses were prepared described previously ${ }^{55}$. Briefly,

424 HEK293T cells were cultured in DMEM supplemented with 10\% fetal bovine serum, L-glutamine,

$425100 \mathrm{U} / \mathrm{ml}$ penicillin and streptomycin and maintained in $5 \% \mathrm{CO}_{2}$ at $37^{\circ} \mathrm{C}$. Briefly, $5 \times 10^{5}$ cells were

426 seeded onto 6-well plates in one day prior to transfection. Cells were co-transfected on the next

427 day at 70-80\% confluency with pLenti-CMV-GFP-Puro $(1.5 \mu \mathrm{g})$ along with pEnvHхв $(0.5 \mu \mathrm{g})$ and

428 psPAX2 $(1 \mu \mathrm{g})$ plasmids. Medium was changed 24h post-transfection. Supernatant was then

429 harvested, filtered with a 0.22 micron filters (Millipore) and titered as described previously ${ }^{55}$. The

430 virus was stored at $-80^{\circ} \mathrm{C}$ until use.

431 SARS-CoV-2 S protein pseudotyped lentiviruses were produced as described by Crawford et al.

$432{ }^{56}$ and the following reagents were obtained through BEI resources, NIAID, NIH: SARS-Related

433 Coronavirus 2, Wuhan-Hu-1 Spike-Pseduotyped Lentiviral Kit, NR-52948. In brief, HEK293T

434 cells seeded in $15 \mathrm{~cm}$ dishes at $1.1 \times 10^{7}$ cells $/ \mathrm{mL}$ in $15 \mathrm{~mL}$ of standard DMEM. 16- 24 hours post 435 seeding, cells were co-transfected with HDM-nCoV-Spike-IDTopt-ALAYT, pHAGE-CMV-

436 Luc2-IRES-ZsGreen-W (BEI catalog number NR-52516), HDM-Hgpm2 (BEI catalog number

437 NR-52517) HDM-tat1b ( BEI catalog number NR-52518), pRC-CMV-Rev1b (BEI catalog NR-

43852519 ). 18-24 hours post transfection the media was replaced with full DMEM. 60 hours post

439 transfection, the supernatant was collected and filtered with a $0.45 \mu \mathrm{m}$ filter and stored at -80

440 degrees. For purification, $40 \mathrm{~mL}$ of supernatant was concentration by spinning at 19, $400 \mathrm{rpm}$ for 
4412 hours. The resulting pellet was resuspended in $400 \mu \mathrm{l}$ of HBSS, followed by 15 mins of

442 continuous vortex at RT. Protein concentration was confirmed by BCA.

443 Coating Polystyrene Microspheres with Protein L and Polyclonal Antibody

444 Fluorescent carboxylate microspheres, $0.5 \mu \mathrm{m}$ (Polysciences) were coated with Protein L

445 (Thermo Scientific), followed by polyclonal IgA or IgG. The polystyrene microspheres were first

446 washed with 1 X PBS and centrifuged at $13523 \mathrm{x}$. The PBS wash was repeated and then the

447 microspheres were incubated at RT with $750 \mu \mathrm{g}$ of Protein L for 4 hours with gentle mixing.

448 Following another PBS wash, $300 \mu \mathrm{g}$ of polyclonal IgA was added to the microspheres and left

449 to incubate at RT with gentle mixing overnight. Following this incubation, the microspheres

450 were centrifuged at $13523 \mathrm{x}$ g for ten minutes, and the resulting pellet was resuspended in $1 \mathrm{~mL}$

451 of PBS and incubated with for 30 minutes with gentle mixing at RT. Following the final

452 incubation, the microspheres were centrifuged at $13523 \mathrm{x}$ g for 5 minutes and resuspended in

$453500 \mu \mathrm{L}$ of PBS.

\section{Neutrophil Stimulation with Soluble ICs}

$45515 \mathrm{~mm}$ glass coverslips were placed inside wells of a sterile 24 -well plate and $4.0 \times 10^{5} \mathrm{PMNs}$

456 were added to each well and allowed to settle for 1 hour. For IAV:polyclonal Ab stimulations,

457 mixtures of $25 \mu \mathrm{g} \mathrm{Cal} / 09$ ( $\left.2^{10} \mathrm{HAU}\right)$ and $50 \mu \mathrm{g} / \mathrm{mL}$ polyclonal IgG or IgA antibody were

458 incubated for 30 minutes at $4^{\circ} \mathrm{C}$ before addition to PMNs. ICs containing monoclonal HA stalk

459 (KB2) or head-binding (29E3) antibodies were generated at a 2:1 ratio of antibody to virus (100

$460 \mu \mathrm{g} / \mathrm{ml}$ and $50 \mu \mathrm{g} / \mathrm{ml}$, respectively) and allowed to incubate for 30 minutes at RT prior to

461 stimulation of PMNs. To test the ability of ICs generated with salivary IgA to stimulate NETosis,

462 matched salivary $\operatorname{IgA}$ and serum $\operatorname{Ig}$ A was purified from the saliva of four healthy donors using

463 peptide M columns. ICs containing $100 \mu \mathrm{g} /$ well of serum derived monomeric IgA or salivary 
$464 \operatorname{IgA}$ and $50 \mu \mathrm{g} /$ well of Cal/09 were allowed to form by incubation at RT 30 minutes. HIV-

465 specific ICs were generated by purifying IgA from the serum of 3 HIV-1 positive donors. ICs

466 were formed by incubating $100 \mu \mathrm{g} / \mathrm{mL}$ of polyclonal IgA and $50 \mu \mathrm{g} / \mathrm{mL}$ of HIV $-1 \mathrm{gp} 120$

467 pseudotyped lentiviruses for 30 minutes at room temperature. SARS-CoV-2 ICs were generated

468 using antibodies purified from the convalescent sera of an individual who had been infected with

469 SARS-CoV-2. ICs were formed by incubating $100 \mu \mathrm{g} / \mathrm{mL}$ of polyclonal IgA and $12.5 \mu \mathrm{g} / \mathrm{mL}$ of

470 pseudotyped spike lentiviruses for 30 minutes at room temperature. For RA samples, immune

471 complexes were formed by incubating $50 \mu \mathrm{g} /$ well of citrullinated human fibrinogen (Cayman

472 Chemicals) with $100 \mu \mathrm{g} /$ well of polyclonal IgA or IgG for 30 minutes at RT. Stimulation of IgA-

473 coated beads was performed by incubating neutrophils with $5.0 \times 10^{8}$ beads. Antibodies were

474 purified from the sera donors diagnosed with RA or healthy donors as described above.

475 Antibodies/viruses/ICs were then incubated with PMNs for 3 hours at $37^{\circ} \mathrm{C}$ before being fixed

476 with 3.7\% paraformaldehyde (PFA) (Pierce Protein Biology) prior to staining and imaging.

\section{Immobilized IC Assay}

478 Purified virus was plated on $15 \mathrm{~mm}$ sterile coverslips in a $24-w e l l$ plate at $2 \mu \mathrm{g} / \mathrm{mL}$ and incubated

479 at $37^{\circ} \mathrm{C}$ for 18 hours. Wells were washed twice with PBS and $250 \mu \mathrm{g}$ of either polyclonal IgA or

$480 \mathrm{IgG}$ was added for 30 minutes at $37^{\circ} \mathrm{C}$. Wells were washed twice with $\mathrm{PBS}$ prior to the addition

481 of $4.0 \times 10^{5} \mathrm{PMNs}$ per well. PMNs were incubated for 3 hours at $37^{\circ} \mathrm{C}$, fixed and stained as

482 described above.

\section{FcoRI Blockade}

$48415 \mathrm{~mm}$ glass coverslips were placed inside wells of a sterile 12 -well plate and $4.0 \times 10^{5} \mathrm{PMNs}$

485 were added to each well in a total volume of $500 \mu \mathrm{L}$ and allowed to settle for 1 hour. To block

486 Fc $\alpha$ RI, $20 \mu \mathrm{g} / \mathrm{mL}$ of mouse anti-human CD89 antibody (AbD Serotec) was added to neutrophils 
487 for 20 minutes at $4^{\circ} \mathrm{C}$. PMNs were then stimulated with various conditions for 3 hours at $37^{\circ} \mathrm{C}$

488 before being fixed with $3.7 \%$ paraformaldehyde (PFA) and stored at $4^{\circ} \mathrm{C}$ until staining.

489 Fluorescence Microscopy and Quantification of NETosis

490 Cells were fixed with 3.7\% PFA (Pierce Protein Biology) at $4^{0} \mathrm{C}$, washed in PBS three times and

491 then permeabilized using 0.5\% Triton X-100 (Thermo Scientific) in PBS-T. Fixed and

492 permeabilized cells were then blocked for 30 minutes at RT in blocking buffer (10\% FBS in

493 PBS-T). Cells were incubated with primary rabbit anti-neutrophil elastase antibody (Abcam) at a

494 1:100 dilution for one hour at room temperature. Coverslips were washed with PBS three times

495 and then incubated with Alexa Fluor 488-conjugated donkey anti-rabbit antibody (Molecular

496 Probes) diluted as per manufacturer's recommendation (2 drops/mL) for 1 hour at RT, protected

497 from light. Coverslips were then washed with PBS three times. $1 \mu \mathrm{g} / \mathrm{mL}$ Hoechst 33342,

498 trihydrochloride, trihydrate (Life Technologies) was incubated for 5 minutes, at RT, protected

499 from light. Cells were washed with PBS three times and coverslips were mounted onto glass

500 slides in EverBrite Mounting Medium (Biotium). Cells were imaged using an EVOS FL

501 microscope (Life Technologies). 5 random fields per condition were captured at 20x

502 magnification. NETosis was quantified by counting cells which had decondensed chromatin

503 colocalized with neutrophil elastase. \% NETosis was expressed as number of cells that had

504 undergone NETosis / number of total cells.

505 Influenza Viral Particle Trapping and Inactivation in NETs

506 Sterilized glass coverslips were placed in a 24 well plate, and neutrophils at $4.0 \times 10^{5}$ cells/well

507 were allowed to settle for 1 hour prior to stimulation. Neutrophils were stimulated with PMA for

5083 hours at $37^{\circ} \mathrm{C}, 5 \% \mathrm{CO}_{2}$. Cal/09 at $10^{5} \mathrm{PFU} / \mathrm{mL}$ was then allowed to settle on the pre-formed

509 NETs for 3 hours at $37^{\circ} \mathrm{C}$, following this incubation cells were fixed with $3.7 \%$ PFA (Pierce 
510 Protein Biology). To stain coverslips for immunofluorescent imaging coverslips were treated in

511 the same was as previously described. Primary antibodies used included primary rabbit anti-

512 neutrophil elastase antibody (Abcam, 1:100 dilution), 6F12 generated from in house-hybridomas

513 at $1 \mathrm{ug} / \mathrm{mL}$. Secondary antibodies included Alexa Fluor 488 conjugated donkey anti-mouse

514 antibody (Molecular Probes, 1:4000) and Alexa Flour 594 donkey anti-rabbit (Molecular Probes,

515 1:4000). Coverslips were incubated with $1 \mu \mathrm{g} / \mathrm{mL}$ Hoechst 33342, trihydrochloride, trihydrate

516 (Life Technologies) to probe for DNA. Cells were visualized and imaged using GFP (Ex 470

$517 \mathrm{~nm} / \mathrm{Em} 525 \mathrm{~nm}$ ) and DAPI (Ex $360 \mathrm{~nm} / \mathrm{Em} 447 \mathrm{~nm}$ ), Texas Red (Ex 585/ Em 624) color cubes

518 in the EVOS FL microscope (Life Technologies). To evaluate inactivation, $10^{5} \mathrm{PFU} / \mathrm{mL}$ of PR8

519 mNeon was incubated with PMA stimulated neutrophils for 3-6 hours. 25 units/mL of DNaseI

520 (Thermo Fisher) was added to PMA stimulated neutrophils and was allowed to incubate for 90

521 minutes to digest NETs. Samples were collected and stored at -80 until further use. Prior to virus

522 quantification, MDCK cells were seeded in 24-well plates and used when 90\% confluent.

523 Sample was diluted 1:10 in 1x minimum essential medium (MEM, Sigma Aldrich) supplemented

524 with 2 mM L-glutamine, 0.24\% sodium bicarbonate, 20 mM HEPES (4-(2-hydroxyethyl)-1-

525 piperazineethanesulfonic acid), MEM amino acids solution (Sigma Aldrich), MEM vitamins

526 solution (Sigma Aldrich), $100 \mathrm{U} / \mathrm{mL}$ penicillin-streptomycin (Thermo Fisher), and 0.42\% bovine

527 serum albumin (Sigma Aldrich), before being added to cells. After 1 hour this was replaced with

528 DMEM containing 10\% FBS (Gibco), 2 mM L-glutamine and $100 \mathrm{U} / \mathrm{mL}$ penicillin-streptomycin

529 (Thermo Fisher). The number of fluorescent cells was assessed 12 hours post-infection. Cells

530 were fixed with PFA and incubated with $1 \mu \mathrm{g} / \mathrm{mL}$ Hoechst 33342, trihydrochloride, trihydrate

531 (Life Technologies). 5-fields per condition were taken on the EVOS FL microscope, and \%

532 infectivity was determined as the number of infected cells / the total number of cells. 


\section{Phagocytosis Assay of Polyclonal Antibody-Coated Microspheres}

534 This protocol was performed as previously described ${ }^{24}$. Briefly, fluorescent carboxylate

535 microspheres $0.5 \mu \mathrm{m}$ (Polysciences) were coated with protein $\mathrm{L}$ and polyclonal $\operatorname{IgA}$ or IgG and

536 were incubated with neutrophils at a $500: 1$ ratio at $37^{\circ} \mathrm{C}$ for 15 minutes with gentle mixing. This

537 was followed by centrifugation at $930 \times \mathrm{g}$ for 10 minutes. Cells were washed twice with PBS

538 before being plated in a 96-well plate. Fluorescence was measured with the SpectraMax i3 plate

539 reader at 526nm (Molecular Devices).

\section{NOX Assay}

541 Neutrophils were purified as described above and allowed to settle on glass coverslips for 1 hour

542 at $37^{\circ} \mathrm{C}$. While settling, neutrophils were incubated with $20 \mu \mathrm{m}$ of Diphenyleneiodonium

543 chloride (DPI) (Sigma-Aldrich) a neutrophil NADPH oxidase inhibitor. Neutrophils were then

544 stimulated with IgA:IAV ICs or PMA $(0.1 \mathrm{mg} / \mathrm{mL}$, Sigma-Aldrich $)$ as a positive control and DPI

545 was maintained in the media. Cells were then fixed with 3.7\% paraformaldehyde (PFA) (Pierce

546 Protein Biology) and stored at $4{ }^{\circ} \mathrm{C}$ until staining and imaging.

\section{Statistics}

548 Graphs and statistical analyses were generated using Graphpad Prism v9 (Graphpad Software,

549 San Diego, CA). A $P$ value of $<0.05$ was considered to be significant across all experiments.

\section{FIGURE LEGENDS}

551 Figure 1. IgA:IAV ICs potentiate NETosis. (A-F) Primary human neutrophils were isolated

552 from the peripheral blood of healthy donors $(n=3$ or 4$)$, and stimulated with PMA, monoclonal or

553 polyclonal IgG or IgA antibodies, or ICs for 3 hours as shown. NETosis was assessed by

554 immunofluorescence microscopy after co-staining for DNA (DAPI) and neutrophil elastase. (A)

555 Representative images are shown (20x). Bars depict $200 \mu \mathrm{m}$. Insert (a) shows area with NETs, 
556 insert (b) shows area with intact neutrophils. (B) The percentage of cells that had undergone

557 NETosis (defined by typically NET morphology and co-staining of DAPI + neutrophil elastase)

558 were quantified in a blinded manner from 5 fields in 4 independent experiments. (C, D) The

559 assay was repeated using monoclonal antibodies, (C) KB2 and (D) 29E3, which bind the HA

560 stalk and head domain of Cal/09, respectively. (E, F) To determine the phenotype of mixed

$561 \mathrm{IgG} / \mathrm{IgA}$ ICs, polyclonal $\mathrm{IgG}$ and $\mathrm{IgA}$ were mixed with $\mathrm{Cal} / 09$ at a $(\mathrm{E})$ 1:1 or $(\mathrm{F})$ at the ratio

562 naturally found in serum. For all experiments, percent NETosis was normalized to unstimulated

563 neutrophils. Three or four independent neutrophil donors were used for each experiment. Means

564 and standard error (SEM) of independent experiments are shown. Statistical significance was

565 determined using one-way ANOVA with Tukey post-hoc test. *,P $<0.05 ; * *, \mathrm{P}<0.01$.

566

567 Figure 2. Potentiation of NETosis by IgA ICs is not an IAV-specific phenomenon. (A)

568 Purified SARS-CoV-2 spike pseudotyped lentivirus was titrated onto primary human neutrophils

569 from healthy donors $(\mathrm{n}=3)$ and incubated for 3 hours prior to staining for DNA (DAPI) and

570 neutrophil elastase. (B) Polyclonal IgA was isolated from serum of a convalescent COVID-19

571 donor and from pre-pandemic donor serum (SARS-CoV-2 seronegative) and incubated with

572 spike pseudotyped-lentivirus to form ICs prior to stimulation of neutrophils isolated from healthy

573 donors $(n=3)$ for 3 hours prior to staining for DNA (DAPI) and neutrophil elastase. (C) ICs were

574 formed with IgA purified from serum of HIV-positive individuals (n=3) and HIV-1 X4 gp120

575 (HxB2) pseudotyped lentivirus. Neutrophils were stimulated for 3 hours prior to staining for

576 DNA (DAPI) and neutrophil elastase. (D) Cells were stimulated with ICs containing IgA purified

577 from the serum of healthy donors $(n=5)$ or RA patients $(n=5)$ in complex with citrullinated

578 fibrinogen. NETosis was quantified in a blinded manner from 5 fields per condition. Mean and 
579 SEM of independent experiments are shown. P-values were determined by one-way ANOVA

580 with Tukey post-hoc test. *, $\mathrm{P}<0.05, * *, \mathrm{P}<0.01$.

Figure 3. IgA ICs induce NETosis via FcoRI engagement, independently of TLR signaling

584 antibody:IAV ICs generated from matched salivary $\operatorname{Ig} \mathrm{A}(\operatorname{sg} \mathrm{A})$ and serum $\operatorname{Ig} \mathrm{A}$ of healthy IAV-

585 exposed donors (n=4). (B) Primary human neutrophils were incubated with an anti-CD89

586 (Fc $\alpha$ RI) antibody prior to stimulation with IgG:IAV or IgA:IAV ICs (n=3) (C) Primary human

587 neutrophils were stimulated with polyclonal IgA, polystyrene beads coated with Protein L, or

588 polystyrene beads coated with protein L and IgA. For all experiments, NETosis was assessed by

589 immunofluorescence microscopy analysis of cells co-stained for DNA (DAPI) and neutrophil

590 elastase. NETosis in stimulated conditions was normalized to untreated cells (n=6). (D)

591 Fluorescent polystyrene beads were coated with protein L, followed by either polyclonal IgG or

$592 \operatorname{Ig}$ A. Human neutrophils were isolated and incubated with the beads at a 500 beads/cell ratio.

593 After washing, phagocytosis of beads was measured using a SpectraMax i3 plate reader

594 (Molecular Devices) (n=3). (E) Purified Cal/09 was immobilized on glass coverslips prior to the

595 addition of IgG or IgA. Primary human neutrophils were added to wells for 3 hours before being

596 fixed and stained for quantification $(n=3)$. Mean and SEM of independent experiments are

597 shown. Statistical significance was evaluated by one-way ANOVA and Tukey post-hoc test. *, P

$598<0.05 ; * *, \mathrm{P}<0.01$

599

600 Figure 4. Influenza virus particles are trapped and inactivated by NETs released via

601 suicidal NETosis. (A) Primary human neutrophils were stimulated with PMA, IgG:IAV, or 
602 IgA:IAV ICs for 30, 90 and 180 minutes prior to fixation and staining for DNA (DAPI) and

603 neutrophil elastase to quantify NETosis. (B) Primary human neutrophils were incubated with

604 DPI, a NOX inhibitor, prior to 3 hours of simulation with IgA:IAV ICs (n=3). (C) Neutrophils

605 were stimulated with PMA for 90 minutes before the addition 105 PFU/ well of IAV. Virus was

606 incubated with the NETs for 3 hours and then fixed and stained with anti-hemagglutinin

607 antibodies (6F12), DNA (DAPI) and neutrophil elastase. Immunofluorescence microscopy was

608 used to measure the co-localization of viral particles (green) with NETs composed of DNA

609 (blue) coated with neutrophil elastase (red) (n=3) (D) Quantification of the raw integrated GFP

610 density was measured using ImageJ and normalized to the number of cells per field. (E)

611 Neutrophils were stimulated with PMA prior to the addition of an IAV expressing an mNeon

612 reporter. Virus was incubated on intact NETs or NETs that had been digested with DNase $(n=3)$.

613 Contents of wells were collected and MDCK cells were infected for 8 hours to measure residual

614 infectivity. Mean and \pm SEM are shown. Statistical significance was evaluated using one-way

615 ANOVA with Tukey post-hoc test. $* \mathrm{P}<0.05 ; * * \mathrm{P}<0.01$

617 Supplementary Figure 1. NETs digested with DNase. Neutrophils were isolated and

618 stimulated with IgA:IAV IC's or PMA for 3 hours. DNase was added at 25 units/mL and

619 allowed to incubate for 90 min prior to fixation and staining.

620 ACKNOWLEDGEMENTS

621 This work was funded by grants from the Canadian Institutes of Health Research (CIHR)

622 (M.S.M.), the Weston Family Microbiome Initiative (M.S.M.), The Lung Association/Ontario

623 Thoracic Society Grants-in-Aid (M.S.M.), and the Michael G. DeGroote Institute for Infectious

624 Disease Research (M.S.M.). M.S.M. was also supporting, in part, by a CIHR New Investigator 
625 Award and an Ontario Early Researcher Award (ERA). H.D.S. was supported, in part, by a

626 CIHR Master's Award and an Ontario Graduate Scholarship. The authors thank Dr. Joe Mymryk

627 for critical reading of the manuscript and helpful suggestions.

\section{AUTHOR CONTRIBUTIONS}

629 M.S.M. and C.E.M. conceived project. H.D.S., D.G, A.P., J.C.A., K.E.N., and M.A.Z. performed

630 experiments and analyzed data. C.K., E.C. and D.M.E.B provided critical reagents and

631 experimental input. H.D.S. and M.S.M. wrote the manuscript with input from all authors.

632

633

634

635

636

637

638

639

640

641

642

643

644

645

646

647

648

649

650

651

652

653

654

655

656

657

658

659

660

661

662

663

\section{REFERENCES}

1. Bakema, J. E. \& van Egmond, M. The human immunoglobulin A Fc receptor Fc $\alpha$ RI: a multifaceted regulator of mucosal immunity. Mucosal Immunol. 4, 612-24 (2011).

2. Kolaczkowska, E. \& Kubes, P. Neutrophil recruitment and function in health and inflammation. Nat. Rev. Immunol. 13, 159-175 (2013).

3. Monteiro, R. C. \& Van De Winkel, J. G. J. IgA Fc receptors. Annu. Rev. Immunol. 21, 177-204 (2003).

4. Papayannopoulos, V. Neutrophil extracellular traps in immunity and disease. Nature Reviews Immunology vol. 18 134-147 (2018).

5. Camp, J. V. \& Jonsson, C. B. A role for neutrophils in viral respiratory disease. Frontiers in Immunology vol. 81 (2017).

6. Brinkmann, V. et al. Neutrophil Extracellular Traps Kill Bacteria. Science (80-. ). 303, 1532-1535 (2004).

7. Hiroki, C. H. et al. Neutrophil Extracellular Traps Effectively Control Acute Chikungunya Virus Infection. Front. Immunol. 10, 3108 (2020).

8. Jenne, C. N. et al. Neutrophils recruited to sites of infection protect from virus challenge by releasing neutrophil extracellular traps. Cell Host Microbe 13, 169-180 (2013).

9. Saitoh, T. et al. Neutrophil extracellular traps mediate a host defense response to human immunodeficiency virus-1. Cell Host Microbe 12, 109-116 (2012).

10. Sung, P. S., Huang, T. F. \& Hsieh, S. L. Extracellular vesicles from CLEC2-activated platelets enhance dengue virus-induced lethality via CLEC5A/TLR2. Nat. Commun. 10, (2019).

11. Cortjens, B. et al. Neutrophil extracellular traps cause airway obstruction during respiratory syncytial virus disease. J. Pathol. 238, 401-411 (2016).

12. Toussaint, M. et al. Host DNA released by NETosis promotes rhinovirus-induced type-2 allergic asthma exacerbation. Nat. Med. 23, 681-691 (2017).

13. Narasaraju, T. et al. Excessive neutrophils and neutrophil extracellular traps contribute to acute lung injury of influenza pneumonitis. Am. J. Pathol. 179, 199-210 (2011).

14. Radermecker, C. et al. Neutrophil extracellular traps infiltrate the lung airway, interstitial, and vascular compartments in severe COVID-19. J. Exp. Med. 217, (2020). 
15. Middleton, E. A. et al. Neutrophil extracellular traps contribute to immunothrombosis in

665

666

667

668

669

670

671

672

673

674

675

676

677

678

679

680

681

682

683

684

685

686

687

688

689

690

691

692

693

694

695

696

697

698

699

700

701

702

703

704

705

706

707

708

709

COVID-19 acute respiratory distress syndrome. Blood 136, 1169-1179 (2020).

16. Barnes, B. J. et al. Targeting potential drivers of COVID-19: Neutrophil extracellular traps. Journal of Experimental Medicine vol. 217 (2020).

17. DiLillo, D. J., Palese, P., Wilson, P. C. \& Ravetch, J. V. Broadly neutralizing antiinfluenza antibodies require Fc receptor engagement for in vivo protection. J. Clin. Invest. (2016) doi:10.1172/JCI84428.

18. DiLillo, D. J., Tan, G. S., Palese, P. \& Ravetch, J. V. Broadly neutralizing hemagglutinin stalk-specific antibodies require $\mathrm{F} c \gamma \mathrm{R}$ interactions for protection against influenza virus in vivo. Nat. Med. 20, 143-51 (2014).

19. He, W. et al. Alveolar macrophages are critical for broadly-reactive antibody-mediated protection against influenza A virus in mice. Nat. Commun. 8, 846 (2017).

20. Bernstein, D. I. et al. Immunogenicity of chimeric haemagglutinin-based, universal influenza virus vaccine candidates: interim results of a randomised, placebo-controlled, phase 1 clinical trial. Lancet Infect. Dis. (2019) doi:10.1016/S1473-3099(19)30393-7.

21. Forthal, D. N. \& Finzi, A. Antibody-dependent cellular cytotoxicity in HIV infection. AIDS (London, England) vol. 32 2439-2451 (2018).

22. He, W. et al. Broadly-Neutralizing Anti-Influenza Virus Antibodies: Enhancement of Neutralizing Potency in Polyclonal Mixtures and IgA Backbones. J. Virol. (2015) doi:10.1128/JVI.03099-14.

23. Tate, M. D. et al. Neutrophils Ameliorate Lung Injury and the Development of Severe Disease during Influenza Infection. J. Immunol. 183, 7441-7450 (2009).

24. Mullarkey, C. E. et al. Broadly neutralizing hemagglutinin stalk-specific antibodies induce potent phagocytosis of immune complexes by neutrophils in an Fc-dependent manner. MBio 7, e01624-16 (2016).

25. Fuchs, T. A. et al. Novel cell death program leads to neutrophil extracellular traps. J. Cell Biol. 176, 231-241 (2007).

26. He, W. et al. Epitope specificity plays a critical role in regulating antibody-dependent cellmediated cytotoxicity against influenza A virus. Proc. Natl. Acad. Sci. 113, 11931-11936 (2016).

27. Leon, P. E. et al. Optimal activation of Fc-mediated effector functions by influenza virus hemagglutinin antibodies requires two points of contact. Proc. Natl. Acad. Sci. U. S. A. 113, (2016).

28. Tan, G. S. et al. A Pan-H1 Anti-Hemagglutinin Monoclonal Antibody with Potent BroadSpectrum Efficacy In Vivo. J. Virol. 86, 6179-6188 (2012).

29. Tan, G. S. et al. Characterization of a Broadly Neutralizing Monoclonal Antibody That Targets the Fusion Domain of Group 2 Influenza A Virus Hemagglutinin. J. Virol. 88, 13580-13592 (2014).

30. Heaton, N. S. et al. In Vivo Bioluminescent Imaging of Influenza A Virus Infection and Characterization of Novel Cross-Protective Monoclonal Antibodies. J. Virol. 87, 82728281 (2013).

31. Hai, R. et al. Influenza Viruses Expressing Chimeric Hemagglutinins: Globular Head and Stalk Domains Derived from Different Subtypes. J. Virol. 86, 5774-5781 (2012).

32. Lood, C., Arve, S., Ledbetter, J. \& Elkon, K. B. TLR7 / 8 activation in neutrophils impairs immune complex phagocytosis through shedding of FcgRIIA. J. Exp. Med. 1-17 (2017).

33. Duchemin, M., Tudor, D., Cottignies-Calamarte, A. \& Bomsel, M. Antibody-Dependent 

HIV-1 Envelope Subunit gp41. Front. Immunol. 11, 1141 (2020).

34. Veras, F. P. et al. SARS-CoV-2 triggered neutrophil extracellular traps (NETs) mediate COVID-19 pathology. J. Exp. Med. 217, (2020).

35. Zuo, Y. et al. Neutrophil extracellular traps in COVID-19. JCI Insight 5, (2020).

36. Aleyd, E., Al, M., Tuk, C. W., van der Laken, C. J. \& van Egmond, M. IgA Complexes in Plasma and Synovial Fluid of Patients with Rheumatoid Arthritis Induce Neutrophil Extracellular Traps via FcoRI. J. Immunol. 197, 4552-4559 (2016).

37. Wright, H. L., Moots, R. J. \& Edwards, S. W. The multifactorial role of neutrophils in rheumatoid arthritis. Nature Reviews Rheumatology vol. 10 593-601 (2014).

38. Hill, J. A., Al-Bishri, J., Gladman, D. D., Cairns, E. \& Bell, D. A. Serum autoantibodies that bind citrullinated fibrinogen are frequently found in patients with rheumatoid arthritis. J. Rheumatol. 33, 2115-2119 (2006).

39. Herr, A. B., Ballister, E. R. \& Bjorkman, P. J. Insights into IgA-mediated immune responses from the crystal structures of human FcaRI and its complex with IgA1-Fc. Nature 423, 614-620 (2003).

40. Heil, F. et al. Species-Specific Recognition of Single-Stranded RNA via Toll-like Receptor 7 and 8. Science (80-. ). 303, 1526-1529 (2004).

41. Yipp, B. G. \& Kubes, P. NETosis: how vital is it? Blood 122, 2784-94 (2013).

42. Harding, A. T., Heaton, B. E., Dumm, R. E. \& Heaton, N. S. Rationally designed influenza virus vaccines that are antigenically stable during growth in eggs. MBio $\mathbf{8}$, (2017).

43. Papayannopoulos, V. \& Zychlinsky, A. NETs: a new strategy for using old weapons. Trends in Immunology vol. 30 513-521 (2009).

44. Zhu, L. et al. High Level of Neutrophil Extracellular Traps Correlates With Poor Prognosis of Severe Influenza A Infection. J. Infect. Dis. (2018) doi:10.1093/infdis/jix475.

45. Jenne, C. N. \& Kubes, P. Virus-Induced NETs - Critical Component of Host Defense or Pathogenic Mediator? PLoS Pathog. 11, 9-12 (2015).

46. Ambrose, C. S., Wu, X., Jones, T. \& Mallory, R. M. The role of nasal IgA in children vaccinated with live attenuated influenza vaccine. Vaccine 30, 6794-6801 (2012).

47. Hoft, D. F. et al. Comparisons of the Humoral and Cellular Immune Responses Induced by Live Attenuated Influenza Vaccine and Inactivated Influenza Vaccine in Adults. (2017) doi:10.1128/CVI.00414-16.

48. Ang, J. C. et al. Comparative Immunogenicity of the 2014-2015 Northern Hemisphere Trivalent IIV and LAIV against Influenza A Viruses in Children. Vaccines 7, 87 (2019).

49. Nachbagauer, R. et al. A chimeric hemagglutinin-based universal influenza virus vaccine approach induces broad and long-lasting immunity in a randomized, placebo-controlled phase I trial. Nat. Med. (2020) doi:10.1038/s41591-020-1118-7.

50. Gonzalez-Quintela, A. et al. Serum levels of immunoglobulins ( $\operatorname{IgG}, \operatorname{IgA}, \operatorname{Ig} M)$ in a general adult population and their relationship with alcohol consumption, smoking and common metabolic abnormalities. Clin. Exp. Immunol. 151, $42-50$ (2008).

51. Duchemin, M., Khamassi, M., Lin, X., Tudor, D. \& Bomsel, M. IgA Targeting Human Immunodeficiency Virus-1 Envelope gp41 Triggers Antibody-Dependent Cellular Cytotoxicity Cross-Clade and Cooperates with gp41-Specific IgG to Increase Cell Lysis. Front. Immunol. 9, (2018).

52. Aleyd, E., Heineke, M. H. \& van Egmond, M. The era of the immunoglobulin A Fc 
receptor FcaRI; its function and potential as target in disease. Immunol. Rev. 268, 123138 (2015).

53. Manicassamy, B. et al. Protection of mice against lethal challenge with 2009 H1N1 influenza A virus by 1918-like and classical swine H1N1 based vaccines. PLoS Pathog. 6, e1000745 (2010).

54. WHO | Manual for the laboratory diagnosis and virological surveillance of influenza. (2011).

55. Zahoor, M. A., Philip, S., Zhi, H. \& Giam, C.-Z. NF- B Inhibition Facilitates the SARS-CoV-2 spike protein for neutralization assays. Viruses 12, 13-15 (2020). 
bioRxiv preprint doi: https://doi.org/10.1101/2021.01.04.424830; this version posted January 10, 2021. The copyright holder for this preprint (which was not certified by peer review) is the author/funder, who has granted bioRxiv a license to display the preprint in perpetuity. It is made available under aCC-BY-NC-ND 4.0 International license.

A

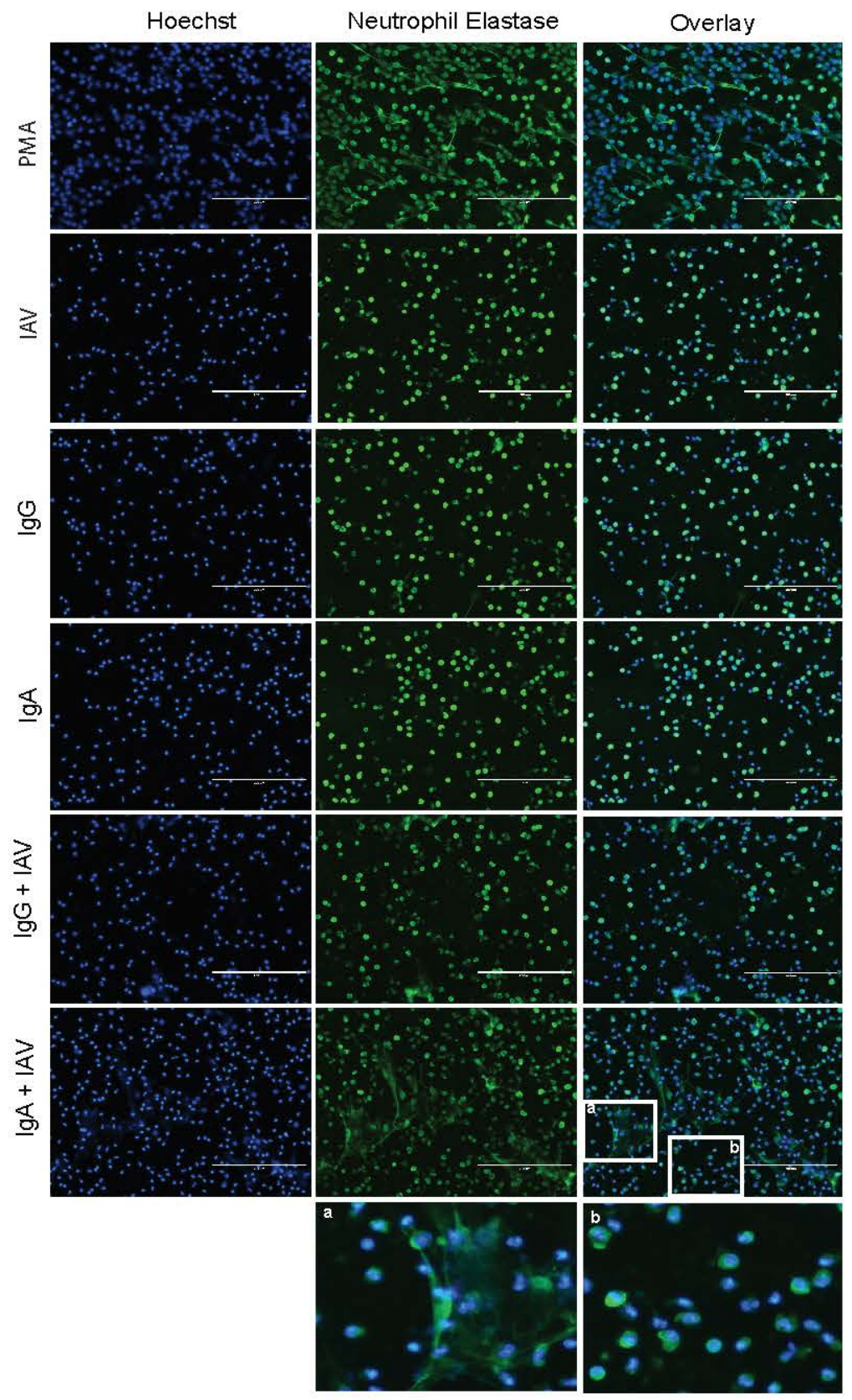

Figure 1. IgA:IAV ICs potentiate NETosis. (A-F) Primary human neutrophils were isolated from the peripheral blood of healthy donors $(n=3$ or 4$)$, and stimulated with PMA, monoclonal or polyclonal IgG or IgA antibodies, or ICs for 3 hours as shown. NETosis was assessed by immunofluorescence microscopy after co-staining for DNA (DAPI) and neutrophil elastase. (A) Representative images are shown (20x). Bars depict $200 \mu \mathrm{m}$. Insert (a) shows area with NETs, insert (b) shows area with intact neutrophils. (B) The $\mathbf{F}$ percentage of cells that had undergone NETosis (defined by typically NET morphology and co-staining of DAPI + neutrophil elastase) were quantified in a blinded manner from 5 fields in 4 independent experiments. (C, D) The assay was repeated using monoclonal antibodies, (C) KB2 and (D) 29E3, which bind the HA stalk and head domain of Cal/09, respectively. (E, F) To determine the phenotype of mixed $\lg \mathrm{G} / \mathrm{IgA} / \mathrm{Cs}$, polyclonal $\lg \mathrm{G}$ and $\lg A$ were mixed with Cal/09 at $a(E) 1: 1$ or $(F)$ at the ratio naturally found in serum. For all experiments, percent NETosis was normalized to unstimulated neutrophils. Three or four independent neutrophil donors were used for each experiment. Means and standard error (SEM) of independent experiments are shown. Statistical significance was determined using one-way ANOVA with Tukey post-hoc test. ${ }^{*}, P<0.05 ;{ }^{* *}, P<0.01$.
B
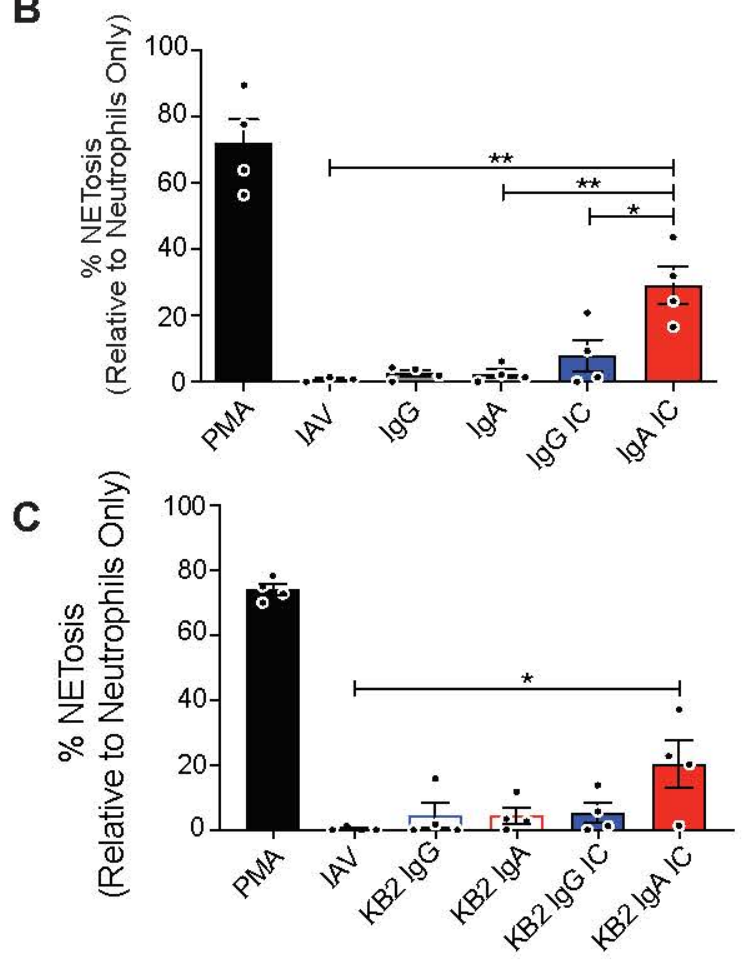

D

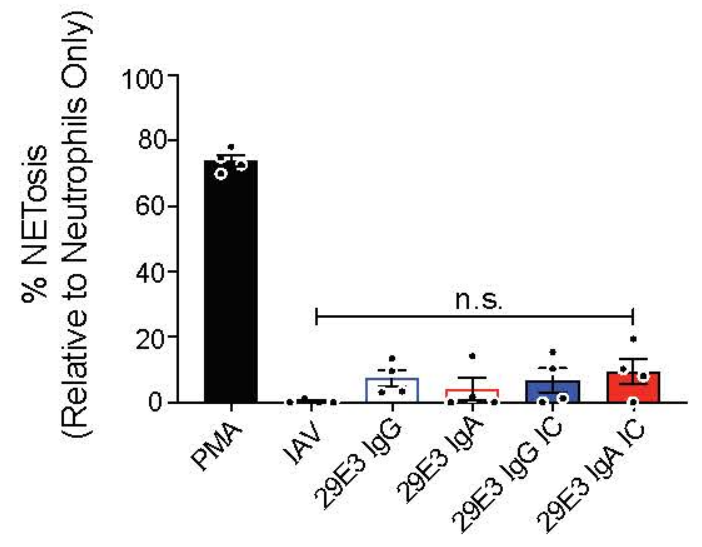

$\mathbf{E}$
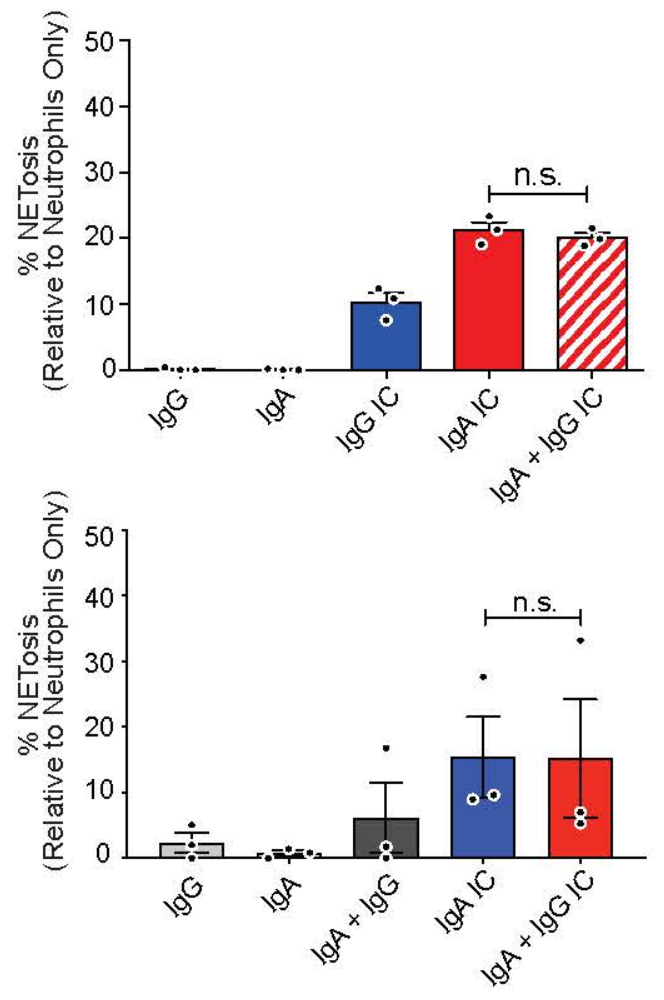
bioRxiv preprint doi: https://doi.org/10.1101/2021.01.04.424830; this version posted January 10,2021 . The copyright holder for this preprint (which was not certified by peer review) is the author/funder, who has granted bioRxiv a license to display the preprint in perpetuity. It is made available under aCC-BY-NC-ND 4.0 International license.

A
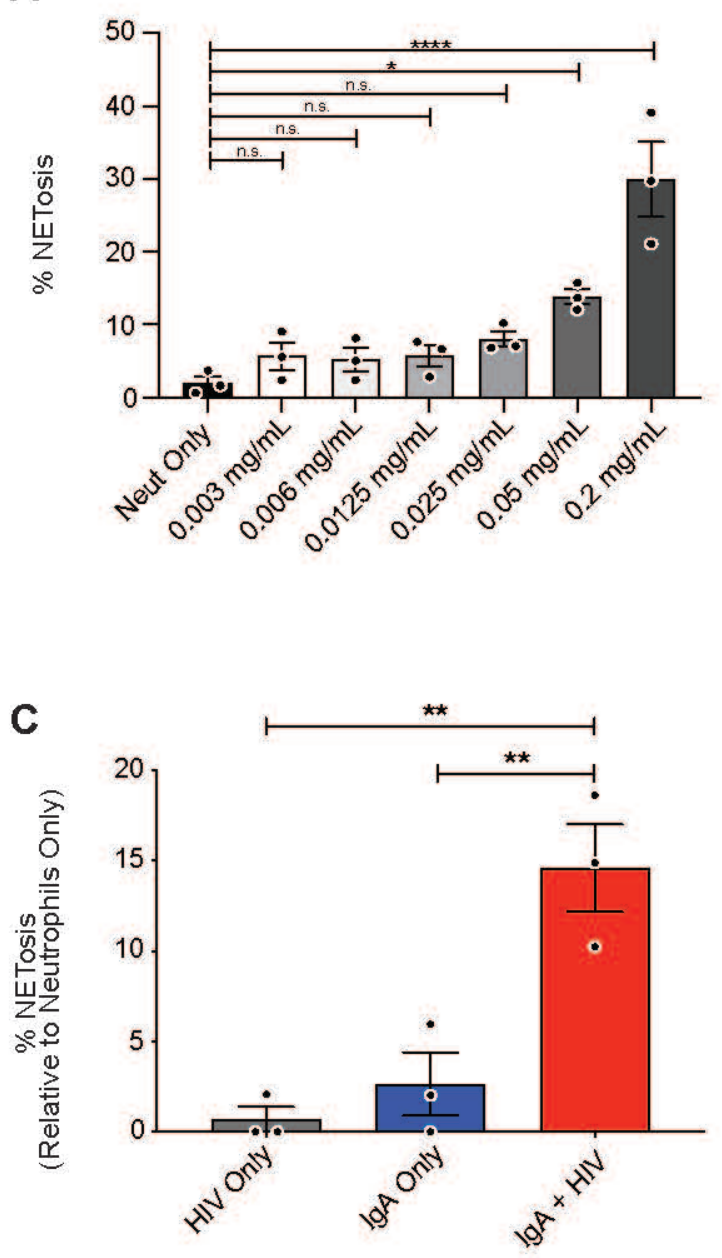

B

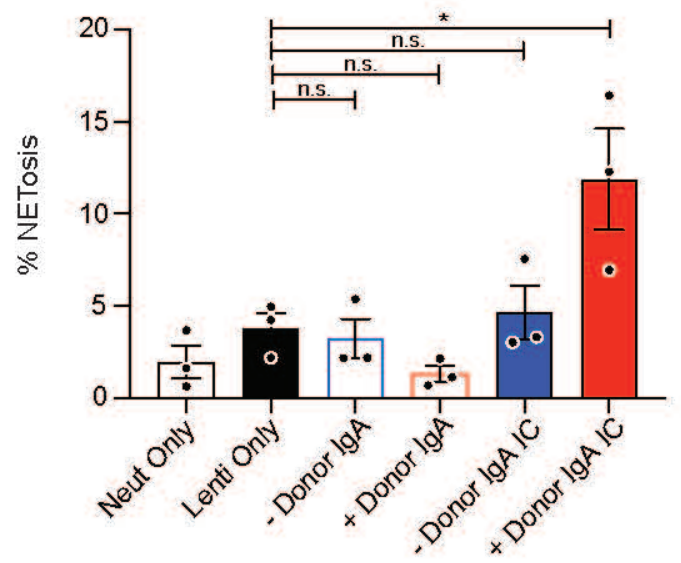

D

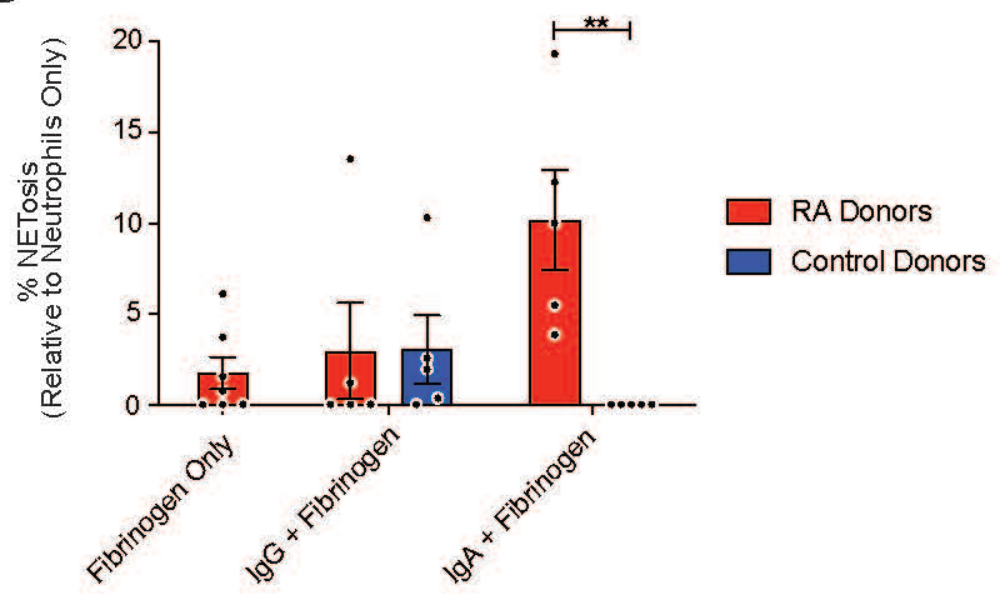

Figure 2. Potentiation of NETosis by IgA ICs is not an IAV-specific phenomenon. (A) Purified SARS-CoV-2 spike pseudotyped lentivirus was titrated onto primary human neutrophils from healthy donors $(n=3)$ and incubated for 3 hours prior to staining for DNA (DAPI) and neutrophil elastase. (B) Polyclonal IgA was isolated from serum of a convalescent COVID-19 donor and from pre-pandemic donor serum (SARS-CoV-2 seronegative) and incubated with spike pseudotyped-lentivirus to form ICs prior to stimulation of neutrophils isolated from healthy donors $(n=3)$ for 3 hours prior to staining for DNA (DAPI) and neutrophil elastase. (C) ICs were formed with IgA purified from serum of HIV-positive individuals ( $n=3$ ) and HIV-1 X4 gp120 (HXB2) pseudotyped lentivirus. Neutrophils were stimulated for 3 hours prior to staining for DNA (DAPI) and neutrophil elastase. (D) Cells were stimulated with ICs containing IgA purified from the serum of healthy donors $(n=5)$ or RA patients $(n=5)$ in complex with citrullinated fibrinogen. NETosis was quantified in a blinded manner from 5 fields per condition. Mean and SEM of independent experiments are shown. P-values were determined by one-way ANOVA with Tukey post-hoc test. ${ }^{*}, P<0.05,{ }^{\star *}, P<0.01$. 
A

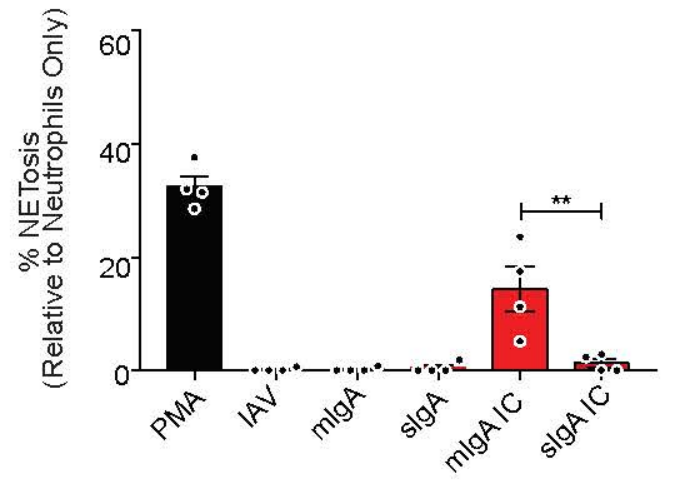

C

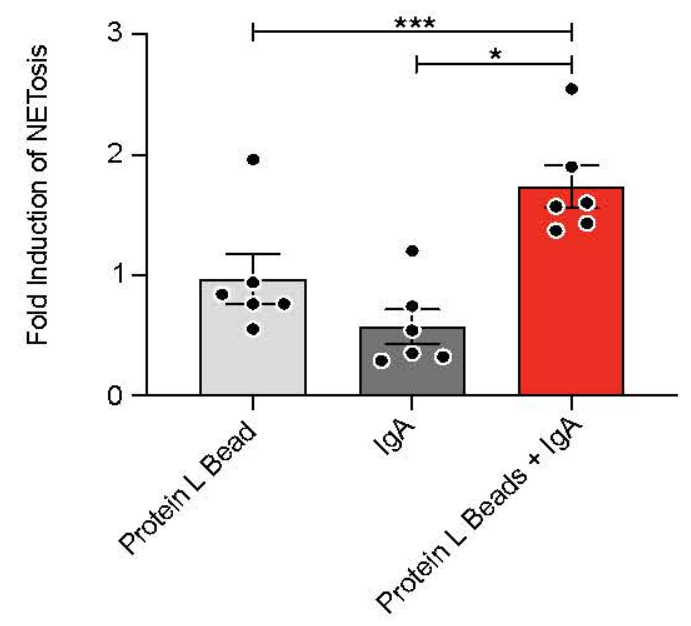

E

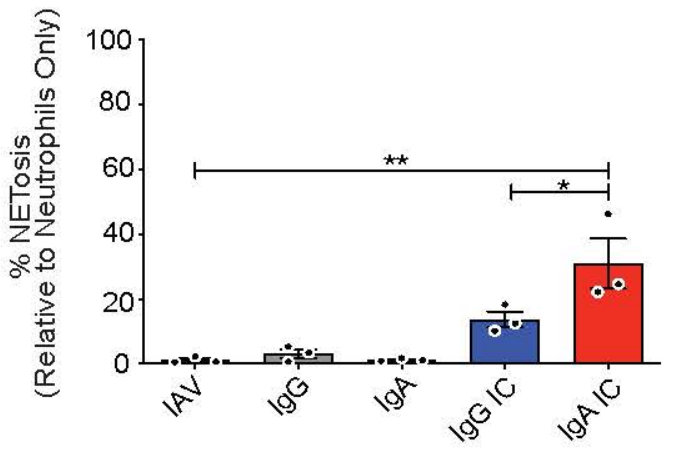

B
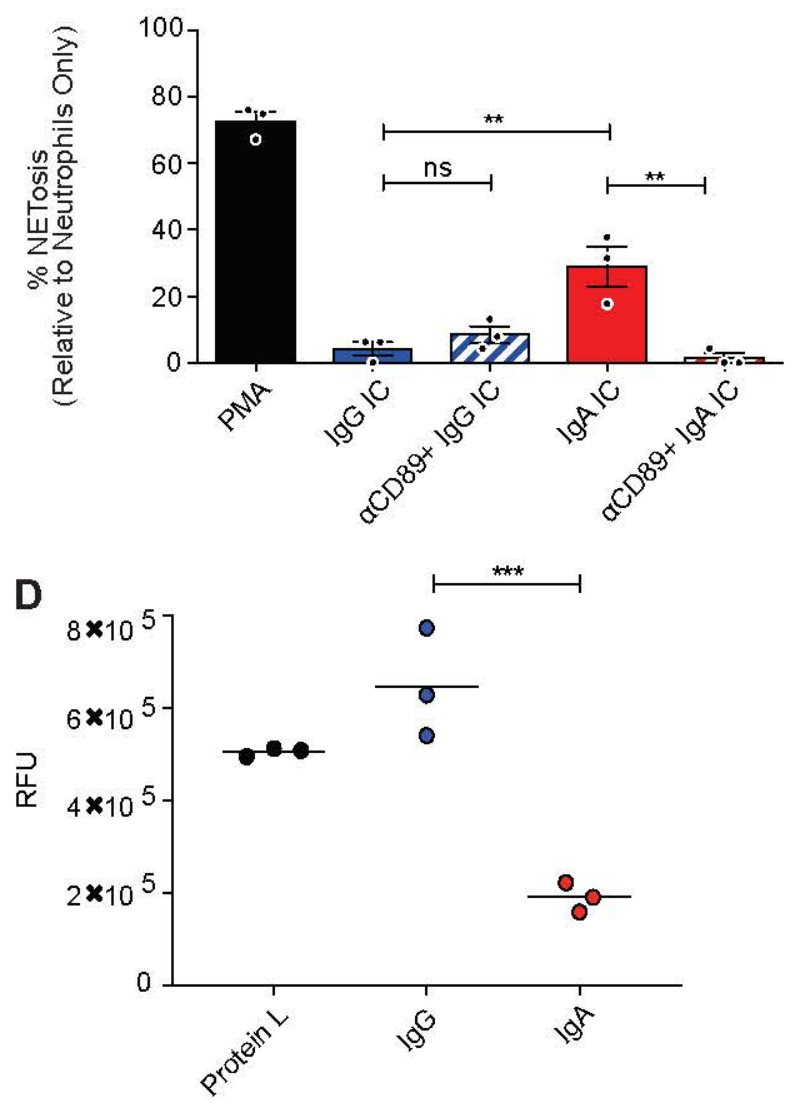

Figure 3. IgA ICs induce NETosis via FcaRI engagement, independently of TLR signaling and phagocytosis. (A) Primary human neutrophils were stimulated for 3 hours with antibody:IAV ICs generated from matched salivary IgA (sIgA) and serum IgA of healthy IAV-exposed donors $(n=4)$. (B) Primary human neutrophils were incubated with an anti-CD89 (FcaRI) antibody prior to stimulation with IgG:IAV or IgA:IAV ICs $(n=3)(C)$ Primary human neutrophils were stimulated with polyclonal IgA, polystyrene beads coated with Protein $L$, or polystyrene beads coated with protein $\mathrm{L}$ and IgA. For all experiments, NETosis was assessed by immunofluorescence microscopy analysis of cells co-stained for DNA (DAPI) and neutrophil elastase. NETosis in stimulated conditions was normalized to untreated cells $(n=6)$. (D) Fluorescent polystyrene beads were coated with protein L, followed by either polyclonal IgG or IgA. Human neutrophils were isolated and incubated with the beads at a 500 beads/cell ratio. After washing, phagocytosis of beads was measured using a SpectraMax i3 plate reader (Molecular Devices) ( $n=3$ ). (E) Purified Cal/09 was immobilized on glass coverslips prior to the addition of IgG or IgA. Primary human neutrophils were added to wells for 3 hours before being fixed and stained for quantification $(n=3)$. Mean and SEM of independent experiments are shown. Statistical significance was evaluated by one-way ANOVA and Tukey post-hoc test. ${ }^{*}, \mathrm{P}<0.05 ;$ **, $\mathrm{P}<0.01$. 
A

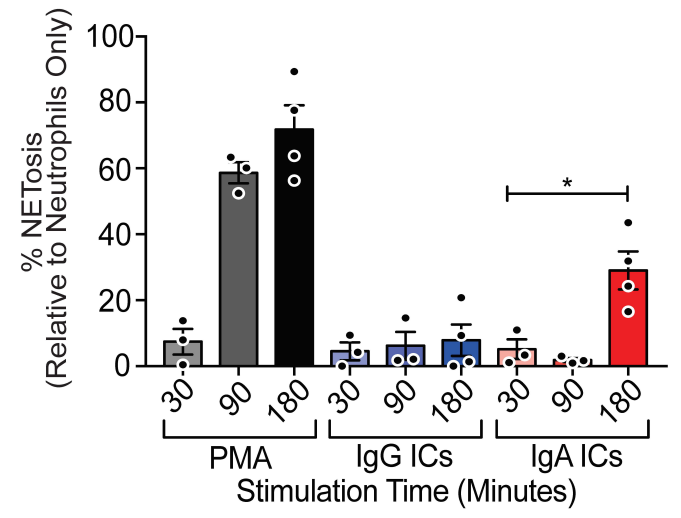

C
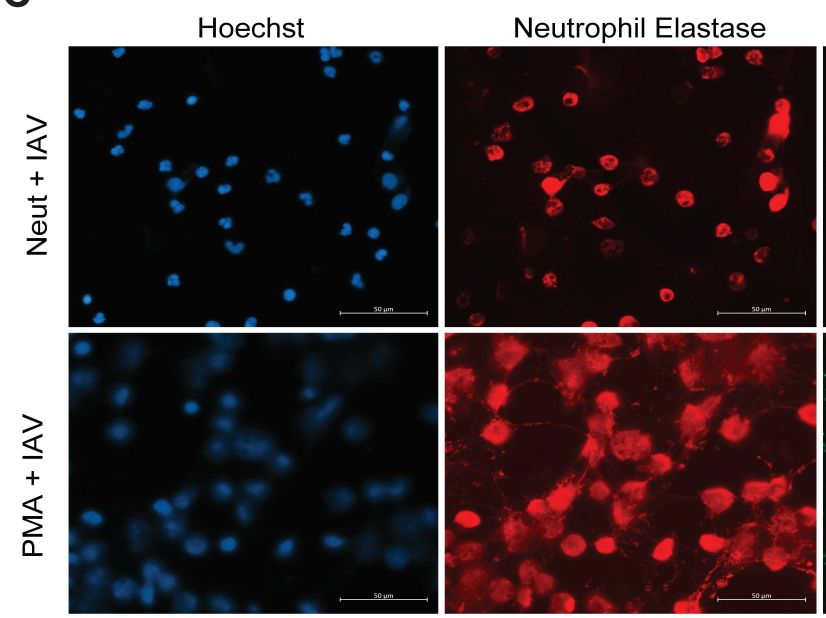

B
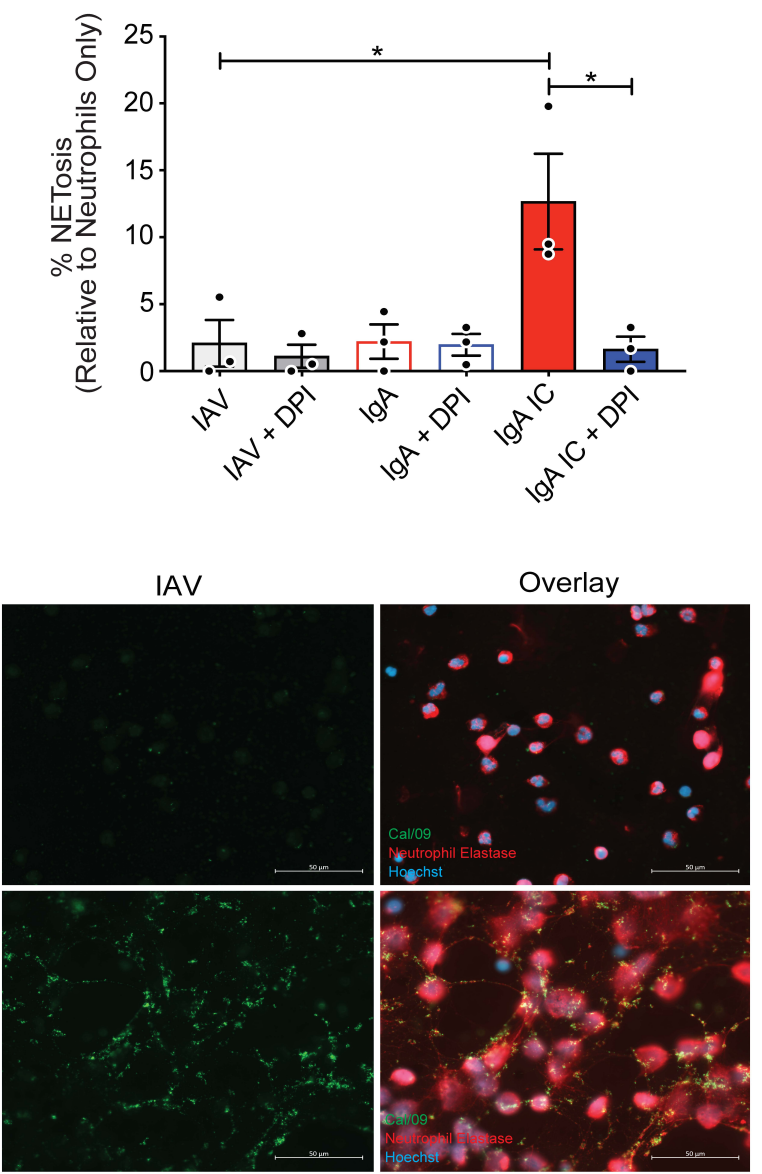

D

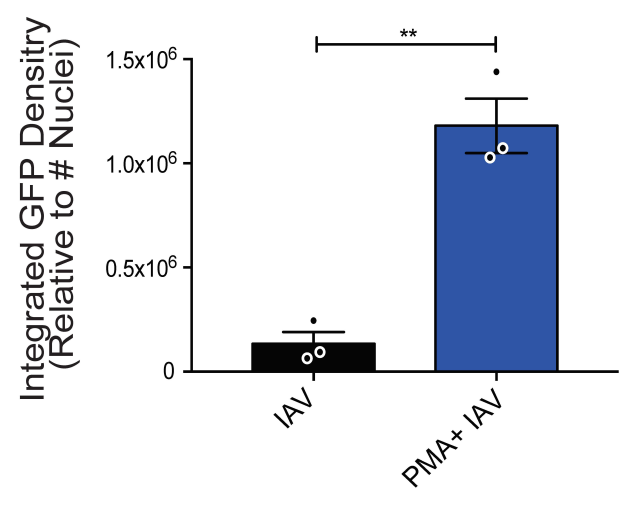

E

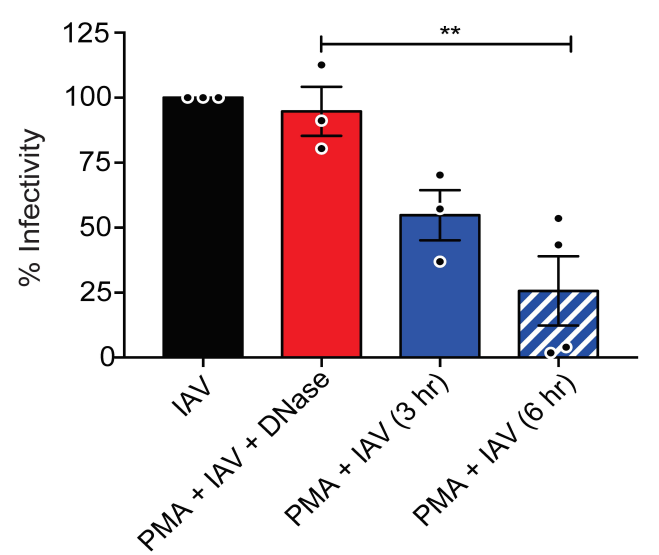

Figure 4. Influenza virus particles are trapped and inactivated by NETs released via suicidal NETosis. (A) Primary human neutrophils were stimulated with PMA, IgG:IAV, or IgA:IAV ICs for 30, 90 and 180 minutes prior to fixation and staining for DNA (DAPI) and neutrophil elastase to quantify NETosis. (B) Primary human neutrophils were incubated with DPI, a NOX inhibitor, prior to 3 hours of simulation with IgA:IAV ICs $(n=3)$. (C) Neutrophils were stimulated with PMA for 90 minutes before the addition 105 PFU/ well of IAV. Virus was incubated with the NETs for 3 hours and then fixed and stained with anti-hemagglutinin antibodies (6F12), DNA (DAPI) and neutrophil elastase. Immunofluorescence microscopy was used to measure the co-localization of viral particles (green) with NETs composed of DNA (blue) coated with neutrophil elastase (red) $(n=3)$ (D) Quantification of the raw integrated GFP density was measured using ImageJ and normalized to the number of cells per field. (E) Neutrophils were stimulated with PMA prior to the addition of an IAV expressing an mNeon reporter. Virus was incubated on intact NETs or NETs that had been digested with DNase $(n=3)$. Contents of wells were collected and MDCK cells were infected for 8 hours to measure residual infectivity. Mean and \pm SEM are shown. Statistical significance was evaluated using one-way ANOVA with Tukey post-hoc test. ${ }^{*} \mathrm{P}<0.05 ;{ }^{*} \mathrm{P}<0.01$. 